\title{
THE SPECTRA OF TOEPLITZ OPERATORS WITH UNIMODULAR SYMBOLS
}

\author{
by TAKAHIKO NAKAZI*
}

(Received 9th January 1996)

\begin{abstract}
The spectrum $\sigma\left(T_{\phi}\right)$ of a Toeplitz operator $T_{\phi}$ on the open unit disc $D$ for a unimodular symbol $\phi$ is studied and many sufficient conditions for $\sigma\left(T_{\phi}\right) \subseteq \partial D$ or $\sigma\left(T_{\phi}\right)=\bar{D}$ are given. In particular if $\phi$ is a unimodular function in $H^{\infty}+C$, then $\sigma\left(T_{\phi}\right) \subseteq \partial D$ or $\sigma\left(\bar{T}_{\phi}\right)=\bar{D}$.
\end{abstract}

1991 Mathematics subject classification: Primary 47B35.

\section{Introduction}

Let $L^{p}$ be the Lebesgue space on the unit circle $\partial D$ and let $H^{p}$ be the corresponding Hardy space for $0<p \leq \infty$. The Toeplitz operator $T_{\phi}$ with symbol $\phi$ in $L^{\infty}$ is the operator on $H^{2}$ defined by $T_{\phi} x=P(\phi x)$ for $x$ in $H^{2}$, where $P$ is the orthogonal projection of $L^{2}$ onto $H^{2}$.

In this paper we study the spectrum $\sigma\left(T_{\phi}\right)$ of a Toeplitz operator $T_{\phi}$. It is known that $\sigma\left(T_{\phi}\right)$ is always connected. This is a hard and deep result due to $\mathrm{H}$. Widom (cf. [2, Corollary 7.46]). If $\phi$ is a continuous function on $\partial D, \sigma\left(T_{\phi}\right)$ consists of the range of $\phi$ together with those points not in the range of $\phi$ that have a nonzero index with respect to $\phi$ (cf. [2, Corollary 7.28]). If $\phi$ is a real-valued function in $L^{\infty}, \sigma\left(T_{\phi}\right)=$ [ess inf $\phi$, ess sup $\phi$ ] (cf. [2, Theorem 7.20]) and if $\phi$ is a function in $H^{\infty}, \sigma\left(T_{\phi}\right)=$ the closure of $\phi(D)$ (cf. [2, Theorem 7.21]). In particular, we are interested in the spectrum $\sigma\left(T_{\phi}\right)$ of a Toeplitz operator $T_{\phi}$ when $\phi$ is a unimodular function in $L^{\infty}$. M. Lee and D. Sarason [6], and R. G. Douglas and D. Sarason [3] have considered $\sigma\left(T_{\phi}\right)$ when $\phi$ is a quotient of two inner functions. Under some conditions, they showed that $\sigma\left(T_{\phi}\right)=\bar{D}[6]$. In this paper, we consider such a problem when $\phi$ is an arbitrary unimodular function. Theorem 1 in [6] is a corollary of (2) of Theorem 2 in this paper. For a real-valued function $s$ in $L^{\infty}, \tilde{s}$ denotes the harmonic conjugate with $\tilde{s}(0)=0$. Our main tool is the following theorem [1].

Widom and Devinatz's Theorem. Let $\phi$ be a unimodular function in $L^{\infty}$. Then the following (1) (3) are equivalent.

* This research was partially supported by Grant-in-Aid for Scientific Research, Ministry of Education. 
(1) $T_{\phi}$ is invertible.

(2) $\phi$ has the form: $\phi=e^{i t}$ where $t$ is a real-valued function in $L^{1}$ such that $\inf \left\{\|t-\tilde{s}-a\|_{\infty} ; s \in L_{R}^{\infty}\right.$ and $\left.a \in R\right\}<\pi / 2$.

(3) $\phi$ has the form: $\phi=g_{1} g_{2} /\left|g_{1} g_{2}\right|$ where both $g_{1}$ and $g_{1}^{-1}$ are in $H^{\infty}$, and both $g_{2}$ and $g_{2}^{-1}$ are in $\bigcup_{p>1} H^{p}$ with $R e g_{2}$ bounded away from 0 on $\partial D$.

In this paper, we give sufficient conditions for $\sigma\left(T_{\phi}\right) \subseteq \partial D$ or $\sigma\left(T_{\phi}\right)=\bar{D}$, using $\inf \|t-\tilde{s}-a\|_{\infty}$ in Section 1 and $g /|g|$ in Section 2. Throughout this paper, for a function space $X$ on $\partial D$, we let $X_{R}=\{\operatorname{Ref} ; f \in X\}$, where Ref is a real part of $f$. C denotes a set of continuous functions on $\partial D$ and so $C_{R}$ is a set of all real valued continuous functions on $\partial D$.

2. Sufficient conditions using inf $\|t-\tilde{s}-a\|_{\infty}$

Lemma 1. Let $\phi$ be unimodular in $L^{\infty}$ and $\lambda=a+i b$ in $D$. Then $\lambda \notin \sigma\left(T_{\phi}\right)$ if and only if $\phi$ has the form $\phi=e^{i t}$ where $t$ is a real-valued function in $L^{1}$ such that

$$
\inf \left\{\left\|t+v_{\lambda}-\tilde{s}-a\right\|_{\infty} ; s \in L_{R}^{\infty} \text { and } a \in R\right\}<\pi / 2
$$

and $v_{\lambda}=\arctan \{(a \sin t-b \cos t) /(1-(a \cos t+b \sin t))\}$.

Proof. We will first show the "if" part. There exists a function $s_{\lambda}$ in $L^{\infty}$ such that $(1-\lambda \bar{\phi}) /|1-\lambda \bar{\phi}|=e^{i s_{\lambda}}$ and $\left\|s_{\lambda}\right\|_{\infty}<\pi / 2$ because $|\lambda|<1$. Then

$$
\frac{1-(a \cos t+b \sin t)}{|1-\lambda \bar{\phi}|}+i \frac{a \sin t-b \cos t}{|1-\lambda \bar{\phi}|}=\cos s_{\lambda}+i \sin s_{\lambda} .
$$

Since $|a \cos t+b \sin t| \leq|\lambda|<1,\left\|v_{\lambda}\right\|_{\infty}<\pi / 2$. Hence $\left\|v_{\lambda}-s_{\lambda}\right\|_{\infty}<\pi$ and $\tan v_{\lambda}=\tan s_{\lambda}$ a.e. and so $v_{\lambda}=s_{\lambda}$ a.e. Therefore

$$
\frac{\phi-\lambda}{|\phi-\lambda|}=\phi \frac{1-\lambda \bar{\phi}}{|1-\lambda \bar{\phi}|}=e^{i t} e^{i \nu_{\lambda}}
$$

and by Widom and Devinatz's Theorem in the Introduction $T_{\phi-2}$ is invertible because $\inf \left\{\left\|t+v_{\lambda}-\tilde{s}-a\right\|_{\infty} ; s \in L_{R}^{\infty}\right.$ and $\left.a \in R\right\}<\pi / 2$. Conversely if $\lambda \notin \sigma\left(T_{\phi}\right)$, by Widom and Devinatz's Theorem there exists a real-valued function $t_{\lambda}$ such that $(\phi-\lambda) /|\phi-\lambda|=e^{i t_{\lambda}}$ and $\inf \left\{\left\|t_{\lambda}-\tilde{s}-a\right\|_{\infty} ; s \in L_{R}^{\infty}\right.$ and $\left.a \in R\right\}<\pi / 2$. As in the proof of the "if" part, there exists $s_{\lambda}$ such that $(1-\lambda \bar{\phi}) /|1-\lambda \bar{\phi}|=e^{i s_{\lambda}}$. Moreover $\phi=e^{i t}$ and $s_{\lambda}=v_{\lambda}$ if $t=t_{\lambda}-s_{\lambda}$. This implies the "only if" part.

Theorem 1. Let $\phi$ be a unimodular function in $L^{\infty}$. 
(1) If $\phi=e^{i t}$ and $t$ is a real-valued function in $L^{1}$ such that inf $\left\{\|t-\bar{s}-a\|_{\infty}\right.$; $s \in L_{R}^{\infty}$ and $a \in R\}=0$, then $\sigma\left(T_{\phi}\right) \subseteq \partial D$.

(2) If $\inf \left\{\|t-\bar{s}-a\|_{\infty} ; s \in L_{R}^{\infty}\right.$ and $\left.a \in R\right\} \geq \pi$ for any $t \in L_{R}^{\prime}$ with $\phi=e^{i t}$, then $\sigma\left(T_{\phi}\right)=\bar{D}$.

(3) If $\sigma\left(T_{\phi}\right)=\bar{D}$, then inf $\left\{\|t-a\|_{\infty} ; a \in R\right\} \geq \pi$ for any $t \in L_{R}^{\prime}$ with $\phi=e^{i t}$.

Proof. (1) If $\lambda=a+i b \in D$ and $v_{\lambda}=\arctan \{(a \sin t-b \cos t) / 1-(a \cos t+b \sin t)\}$, then $\left\|v_{\lambda}\right\|_{\infty}<\pi / 2$ and hence $\inf \left\{\left\|t+v_{\lambda}-\tilde{s}-a\right\|_{\infty} ; s \in L_{R}^{\infty}\right.$ and $\left.a \in R\right\}<\pi / 2$ because $\inf \left\{\|t-\tilde{s}-a\|_{\infty} ; s \in L_{R}^{\infty}\right.$ and $\left.a \in R\right\}=0$. By Lemma $1, \lambda \notin \sigma\left(T_{\phi}\right)$ and hence $\sigma\left(T_{\phi}\right) \subseteq \partial D$.

(2) If $\lambda \in D$ and $\lambda \notin \sigma\left(T_{\phi}\right)$, then by Lemma 1 inf $\left\{\left\|t+v_{\lambda}-\tilde{s}-a\right\|_{\infty} ; s \in L_{R}^{\infty}\right.$ and $a \in R\}<\pi / 2$. Since $\left\|v_{\lambda}\right\|_{\infty}<\pi / 2$, inf $\left\{\|t-\tilde{s}-a\|_{\infty} ; s \in L_{R}^{\infty}\right.$ and $\left.a \in R\right\}<\pi$. This implies (2).

(3) (3) is a result of a theorem of A. Brown and P. R. Halmos (cf. [2, Corollary 7.19]).

Corollary 1. Suppose $\phi=e^{i t}$ and $t$ is a real-valued function which satisfies one of the following (i)-(iii), then $\sigma\left(T_{\phi}\right) \subseteq \partial D$.

(i) $t=\tilde{u}+v$ where $u \in L_{R}^{\infty}$ and $v \in C_{R}$.

(ii) $t=\tilde{u}+v$ where $u \in L_{R}^{\infty}$ and $v$ is in the norm closure of $H_{R}^{\infty}$.

(iii) $t=\tilde{u}+v$ where $u \in L_{R}^{\infty}$ and $v=s \circ q$ for $s \in C_{R}$ and an inner function $q$.

Proof. If $v \in C_{R}$, then $v$ is in the norm closure of $H_{R}^{\infty}$ and so (i) is a result of (ii). If $v \in H_{R}^{\infty}$, then $v=\tilde{s}+a$ for $s \in H_{R}^{\infty}$ and $a \in R$, and hence a simple computation implies (ii). If $s$ is a real-valued polynomial of $z$ and $\bar{z}$, then $v=s \circ q$ belongs to $H_{R}^{\infty}$ for an inner function $q$. Thus (iii) is a result of (ii).

Corollary 2. Let $Q_{j}$ be a non-constant inner function, $a_{j} \in D$ and $b_{j} \in D$ for $1 \leq j \leq \max (n, m)$. Suppose $\phi=\bar{q}_{1} q_{2}$ where $q_{1}=\prod_{j=1}^{n}\left(Q_{j}-a_{j}\right) /\left(1-\bar{a}_{j} Q_{j}\right)$ and $q_{2}=$ $\prod_{j=1}^{m}\left(Q_{j}-b_{j}\right) /\left(1-\bar{b}_{j} Q_{j}\right)$. Then $\sigma\left(T_{\phi}\right) \subseteq \partial D$ if and only if $n=m$.

Proof. If $n=m$, put $u=2 \sum_{j=1}^{n} \log \left|\left(1-\bar{a}_{j} Q_{j}\right) /\left(1-\bar{b}_{j} Q_{j}\right)\right|$, then $u \in L_{R}^{\infty}$ and $\phi=\bar{q}_{1} q_{2}=\alpha e^{\mathrm{i} \overline{\mathrm{j}}}$ for some constant $\alpha$. (1) of Theorem 1 implies the corollary. Suppose $\sigma\left(T_{\phi}\right) \subseteq \partial D$. If $n>m$, then $\phi=\bar{q}_{1} q_{2}=\phi_{1} \phi_{2}$ where $\phi_{1}=\prod_{j=m+1}^{n}\left(1-\bar{a}_{j} Q_{j} / Q_{j}-a_{j}\right)$, $\phi_{2}=\alpha e^{i \bar{i}}, \alpha$ is a constant and $u=2 \sum_{j=1}^{m} \log \left|\left(1-\bar{a}_{j} Q_{j}\right) /\left(1-\bar{b}_{j} Q_{j}\right)\right|$. Therefore $T_{\phi}=T_{\phi_{1}} T_{\phi_{2}}$, and both $T_{\phi}$ and $T_{\phi_{2}}$ are invertible. This contradicts the fact that $T_{\phi_{1}}$ is not invertible. 
3. Sufficient conditions using $g /|g|$ for $g$ in $H^{p}$

Theorem 2. Let $\phi$ be a unimodular function in $L^{\infty}$.

(1) If $\phi=g /|g|$ where both $g$ and $g^{-1}$ are in $H^{\infty}$, then $\sigma\left(T_{\phi}\right) \subseteq \partial D$.

(2) If $\phi \neq g /|g|$ for any $g$ in $\bigcup_{p>1 / 2} H^{p}$ whose inverse is in $\bigcup_{p>1 / 2} H^{p}$, then $\sigma\left(T_{\phi}\right)=\bar{D}$.

Proof. (1) This is a corollary of (1) of Corollary 1 . But we will give another proof. If $\phi=g /|g|$ where both $g$ and $g^{-1}$ are in $H^{\infty}$, put $h=g^{1 / 2}$, then $\phi=h / \bar{h}$ and both $h$ and $h^{-1}$ are in $H^{\infty}$. For any $\lambda \in D, \phi-\lambda=(1 / \bar{h})(1-\lambda \bar{h} / h) h$ and hence

$$
T_{\phi-\lambda}=T_{(1 / \bar{h})} T_{(1-\lambda \bar{h} / h)} T_{h}
$$

This implies that $T_{\phi-\lambda}$ is invertible by Widom and Devinatz's Theorem.

(2) For any $\lambda \in D, 1-\lambda \bar{\phi}=\phi_{0} \ell$ where $\left|\phi_{0}\right|=1$ a.e., and both $\ell$ and $\ell^{-1}$ are in $H^{\infty}$. Hence

$$
\phi-\lambda=\phi(1-\lambda \bar{\phi})=\phi \phi_{0} \ell \text { and } \bar{\phi}_{0}-\ell=\lambda \bar{\phi}_{0} \bar{\phi}
$$

Since $\left\|\bar{\phi}_{0}-\ell\right\|_{\infty}=|\lambda|<1$, by Widom and Devinatz's Theorem $T_{\bar{\phi}_{0}}$ is invertible and $\bar{\phi}_{0}=h /|h|$ for some $h \in H^{a}$ and $a>1$. If $T_{\phi-\lambda}$ is invertible, then $T_{\phi \phi_{0}}$ is invertible and hence $\phi \phi_{0}=k /|k|$ for some $k \in H^{b}$ and $b>1$. Therefore $\phi=\bar{\phi}_{0} \phi \phi_{0}=h k /|h k|$ and both $h k$ and $(h k)^{-1}$ belong to $H^{p}$ for some $p>1 / 2$. This implies (2).

Corollary 3. If $\phi=g /|g|$ where $g \in \bigcap_{p<\infty} H^{p}$ and $g^{-1} \notin \bigcap_{p>1 / 2} H^{p}$, then $\sigma\left(T_{\phi}\right)=\bar{D}$.

Proof. If $\phi=h /|h|$ for some $h$ in $\bigcap_{p>1 / 2} H^{p}$ whose inverse is in $\bigcap_{p>1 / 2} H^{p}$, then $\phi=|k| / k$ with $k=1 / h$. Hence $k g$ is non-negative a.e. on $\partial D$ and $k g \in H^{1 / 2}$. By [7], $g=c h$ for some positive constant $c$ and $g^{-1} \in \bigcap_{p>1 / 2} H^{p}$. Now (2) of Theorem 2 implies the corollary.

Corollary 4. Let $Q_{j}$ be $a$ non-constant inner function, $a_{j} \in D$ and $b_{j} \in D$ for $1 \leq j \leq \max (n, m)$. Suppose $\phi=\bar{q}_{1} q_{2}$ where $q_{1}=\prod_{j=1}^{n}\left(Q_{j}-a_{j}\right) /\left(1-\bar{a}_{j} Q_{j}\right)$ and $q_{2}=$ $\prod_{j=1}^{m}\left(Q_{j}-b_{j}\right) /\left(1-\bar{b}_{j} Q_{j}\right)$. Then $\sigma\left(T_{\phi}\right)=\bar{D}$ if and only if $n \neq m$.

Proof. By Corollary 2, it is enough to show the "if" part. If $n>m$, then by the proof of Corollary $2 \phi=\phi_{2} \phi_{2}$ and so $\phi=\phi_{1}(g /|g|)$ where both $g$ and $g^{-1}$ are in $H^{\infty}$, and $\phi_{1}$ is a non-constant inner function. If $\phi=h /|h|$ for some $h$ in $\bigcap_{p>1 / 2} H^{p}$ whose inverse is in $\bigcap_{p>1 / 2} H^{p}, \phi_{1} g h^{-1}$ is a non-negative function in $H^{1 / 2}$. By [7], this contradicts that $\phi_{1}$ is non-constant. Thus (2) of Theorem 2 implies that $\sigma\left(T_{\phi}\right)=\bar{D}$. When $n<m$, by a similar method we can show that $\sigma\left(T_{\phi}\right)=\bar{D}$.

Now using (2) of Theorem 2, we will give a proof of Theorem 1 in [6]. For each 
inner function $q$, sing $q$ denotes the subset of $\partial D$ on which $q$ can not be analytically extended.

Corollary 5 ([6]). If $\phi=\bar{q}_{1} q_{2}$ where $q_{1}$ and $q_{2}$ are inner functions with sing $q_{1} \neq$ sing $q_{2}$, then $\sigma\left(T_{\phi}\right)=\bar{D}$.

Proof. By (2) of Theorem 2, it is enough to show that $\phi=\bar{q}_{1} q_{2} \neq g /|g|$ for any $g$ in $\bigcap_{p>1 / 2} H^{p}$ whose inverse is in $\bigcap_{p>1 / 2} H^{p}$. We may assume that sing $q_{1} \not \supset z_{0} \in \operatorname{sing} q_{2}$. There exists a constant $\lambda \in D$ such that $q=\left(q_{2}-\lambda\right) /\left(1-\bar{\lambda} q_{2}\right)$ is a Blaschke product with sing $q=\operatorname{sing} q_{2}$ by [5, p. 176]. Then $\bar{q}_{1} q_{2}=\bar{q}_{1} q k /|k|$ where $k=\left(1-\bar{\lambda} q_{2}\right)^{2}$. Since both $k$ and $k^{-1}$ are in $H^{\infty}$, we may assume that $q_{2}$ is a Blaschke product. If $\bar{q}_{1} q_{2}=f /|f|$ $q_{1} \bar{q}_{2}=g /|g|$ where $f g=1$ a.e., $f \in H^{1 / 2}$ and $g \in H^{1 / 2}$, then $\bar{q}_{1} q_{2} g \geq 0$ a.e. and $\bar{q}_{2} q_{1} f \geq 0$ a.e. Since $\bar{q}_{1} q_{2} g \geq 0$ a.e., $g \in H^{1 / 2}$ and $z_{0} \notin$ sing $q_{1}$, by [4] there exists an open arc $J$ such that $z_{0} \in J$ and $q_{2} g$ can be continued analytically from $D$ across $J$. The zeros of $q_{2}$ cannot cluster at any point of $J$. This contradicts that $z_{0} \in \operatorname{sing} q_{2}$. Thus $\bar{q}_{1} q_{2}$ satisfies the condition of (2) of Theorem 2, and hence $\sigma\left(T_{\phi}\right)=\bar{D}$.

Corollary 6. Let $q_{1}$ and $q_{2}$ be inner functions, and $\chi_{E}$ be a characteristic function of a measurable set $E$ in $\partial D$. If $\phi=\bar{q}_{1} q_{2}\left(2 \chi_{E}-1\right)$ and there exists an open arc $J$ in $E$ such that (sing $\left.q_{2}\right) \cap J \neq \emptyset$ and (sing $\left.q_{1}\right) \cap J=\emptyset$, or (sing $\left.q_{1}\right) \cap J \neq \emptyset$ and (sing $\left.q_{2}\right) \cap J=\emptyset$, then $\sigma\left(T_{\phi}\right)=\bar{D}$.

Proof. As in Corollary 5, we may assume that $q_{2}$ is a Blaschke product. If $\phi=\vec{q}_{1} q_{2}\left(2 \chi_{E}-1\right)=f /|f|=|g| / g$ where $f g=1$ a.e., $f \in H^{1 / 2}$ and $g \in H^{1 / 2}$, then $\bar{q}_{1} q_{2}\left(2 \chi_{E}-1\right) g \geq 0$ a.e. and $\bar{q}_{2} q_{1}\left(2 \chi_{E}-1\right) f \geq 0$ a.e.. If there exists an open arc $J$ in $E$ such that (sing $\left.q_{2}\right) \cap J \neq \emptyset$ and (sing $q_{1}$ ) $\cap J=\emptyset$, then

$$
\bar{q}_{1} q_{2}\left(2 \chi_{E}-1\right) g=\bar{q}_{1} q_{2} g \geq 0 \text { a.e. on } J \text {. }
$$

Now as in Corollary 5 , we can get a contradiction and hence $\sigma\left(T_{\phi}\right)=\bar{D}$.

Let $q_{a}=\exp \{-a(1+z) /(1-z)\}$ for $a>0$ and suppose $b$ is a Blaschke product with $\operatorname{sing} b=\{1\}$. Put $\phi_{a}=\bar{q}_{a} b$. Theorem 4 in [6] shows that if $\phi_{a}$ belongs to $H^{\infty}+C$ for all $a>0$, then $\sigma\left(T_{\phi_{s}}\right)=\bar{D}$. This is a corollary of Corollary 7 .

Corollary 7. If $\phi_{a}$ belongs to $H^{\infty}+C$ for some $a>0$, then $\sigma\left(T_{\phi_{c}}\right)=\bar{D}$ for $0<c<a$. If $T_{\phi_{\mathrm{e}}}$ is invertible or $\sigma\left(T_{\phi_{\mathrm{s}}}\right) \subseteq \partial D$, then $\sigma\left(T_{\phi_{\mathrm{c}}}\right)=\bar{D}$ for arbitrary $c>0$ with $c \neq a$.

Proof. By Theorem 2 in [8], $\phi_{a}=q e^{(u+t)}$ where $q$ is inner, and $u$ and $v$ are in $C_{R}$. For $0<c<a, \phi_{c}=q_{a-c} q e^{(u+t)}$ and so by (2) of Theorem $2 \sigma\left(T_{\phi_{c}}\right)=\bar{D}$. For if $q_{a-c} q e^{q(u+\bar{v})}=g /|g|$ for some $g$ in $\bigcup_{p>1 / 2} H^{p}$ with $h=g^{-1} \in \bigcup_{p>1 / 2} H^{p}$, then $h q_{a-c} q e^{i(u+\tilde{\theta})} \geq 0$ a.e. and so $h k q_{a-c} q \geq 0$ a.e. where $k=e^{-\bar{u}+0+(u+t)}$. Since both $k$ and $k^{-1}$ belong to $\bigcap_{p<\infty} H^{p}, h k q_{a-\varepsilon} q$ is a non-negative function in $H^{1 / 2}$ and so by [7], $h k q_{a-\varepsilon} q$ is constant. 
This contradicts the fact that $q_{a-c} q$ is not constant. Therefore (2) of Theorem 2 shows that $\sigma\left(T_{\phi_{c}}\right)=\bar{D}$ for $0<c<a$. If $T_{\phi_{o}}$ is invertible, it is known that $q$ is constant. In fact, we can show it as in the above proof. If $q$ is constant, then for $c>0$ with $c \neq a$

$$
\phi_{c}=\bar{q}_{c} b=q_{a-c} \bar{q}_{a} b=q_{a-c} e^{i(u+\bar{v})} .
$$

By the first part of this theorem, we may assume that $c>a$. However, in this case we can show it as in case $0<c<a$.

\section{Remark}

If $\sigma\left(T_{\phi}\right) \subseteq \partial D$, then $\sigma\left(T_{\phi}\right)=J$ for some closed arc $J$ in $\partial D$ because $\sigma\left(T_{\phi}\right)$ is connected by a theorem of $\mathrm{H}$. Widom (cf. [2, Corollary 7.46]). Then, if the essential range $R(\phi)$ of $\phi$ is disconnected, by a theorem of A. Brown and P. R. Halmos (cf. [2, Corollary 7.19]), then $\sigma\left(T_{\phi}\right) \notin \partial D$. Hence if $\sigma\left(T_{\phi}\right) \subseteq \partial D, R(\phi)$ is connected and so $R(\phi)=J=\sigma\left(T_{\phi}\right)$ by the theorem of A. Brown and P. R. Halmos. If $\phi=\alpha e^{i t}$, $\inf \left\{\|t-\tilde{s}\|_{\infty} ; s \in L_{R}^{\infty}\right\}=0$ and $R(\phi)=\partial D$, then $\sigma\left(T_{\phi}\right)=\partial D$ by (1) of Theorem 1. For a unimodular function $\phi$ in $C$, by Theorem 1 it is easy to see that $\sigma\left(T_{\phi}\right) \subseteq \partial D$ if and only if $\phi=e^{i v}$ for some $v \in C_{R}$. For a unimodular function $\phi$ in $H^{\infty}+C$, by [8, Theorem 2] and Theorem 1 it is easy to see that $\sigma\left(T_{\phi}\right) \subseteq \partial D$ if and only if $\phi=e^{i(\hat{u}+v)}$ for some $u, v \in C_{R}$. In fact, by [8, Theorem 2], Theorems 1 and $2, \sigma\left(T_{\phi}\right) \subseteq \partial D$ or $\sigma\left(T_{\phi}\right)=\bar{D}$ for a unimodular function $\phi$ in $H^{\infty}+C$.

In Corollary 3, we can not change the condition: $g^{-1} \notin \bigcup_{p>1 / 2} H^{p}$ to $g^{-1} \notin \bigcup_{p>1} H^{p}$ even if $g \in H^{\infty}$. For example, put $g=1+z$ then $\sigma\left(T_{\phi}\right) \neq \bar{D}$. If $\phi=(1+q)^{\alpha} /|1+q|^{\alpha}$ where $q$ is a non-constant inner function and $2 \leq \alpha<\infty$, then by Corollary 3 $\sigma\left(T_{\phi}\right)=\bar{D}$ because $(1+q)^{\alpha} \in H^{\infty}$ and $(1+q)^{-\alpha} \notin \bigcup_{p>1 / 2} H^{p}$. We can show a more general theorem than Corollary 6 , that is, for a symbol $\phi=\bar{q}_{1} q_{2} \phi_{0}$ where $\phi_{0}$ is a unimodular step function. Let $\phi$ be an arbitrary unimodular function in $L^{\infty}$, then by [8] $\phi=\bar{q}_{1} q_{2} e^{i(u+\tilde{v})}$ where both $q_{1}$ and $q_{2}$ are Blaschke products and $u, v \in C_{R}$. If $\operatorname{sing} q_{1} \neq$ sing $q_{2}$, then by the proof of Corollary 5 it is easy to see that $\phi \neq g /|g|$ for any $g$ in $\bigcap_{p>1 / 2} H^{p}$ whose inverse is in $\bigcap_{p>1 / 2} H^{p}$. Thus by Theorem $2 \sigma\left(T_{\phi}\right)=\bar{D}$.

\section{REFERENCES}

1. A. Devinatz, Toeplitz operator on $H^{2}$ spaces, Trans. Amer. Math. Soc. 112 (1964), 304 317.

2. R. G. Douglas, Banach algebra techniques in operator theory (Academic Press, New York, 1972).

3. R. G. Douglas and D. E. Sarason, A class of Toeplitz operators, Indiana U. Math. J. 20 (1971), 891-895. 
4. T. W. Gamelin, J. B. Garnett, L. A. Rubel and A. L. Shields, On badly approximable functions, J. Approx. Theory 17 (1976), 280-296.

5. K. Hoffman, Banach spaces of analytic functions (Prentice-Hall, Englewood Cliffs, New Jersey, 1962).

6. M. Lee and D. E. Sarason, The spectra of some Toeplitz operators, J. Math. Anal. Appl. 33 (1971), 529-543.

7. J. Neuwirth and D. J. Newman, Positive $H^{1 / 2}$ functions are constant, Proc. Amer. Math. Soc. 18 (1967), 958.

8. T. H. WolfF, Two algebras of bounded functions, Duke Math. J. 49 (1982), 321-328.

DepartMENT OF MATHEMATICS

Faculty OF SCIENCE

HOKKAIDO UNIVERSITY

SAPPORO 060

JAPAN 